\title{
Position effects in comparative judgments of serial order: List structure vs. differential strength
}

\author{
WILLIAM P. BANKS \\ Pomona College and Claremont Graduate School, Claremont, California 91711 \\ HEDY WHITE \\ Claremont Graduate School, Claremont, California 91711 \\ and \\ ROBIN MERMELSTEIN \\ University of Oregon, Eugene, Oregon 97403
}

\begin{abstract}
Two experiments are reported that test the hypothesis that the serial position effect in comparative judgment of ordinal position in arbitrary serial lists results from differential memory or associative strength among list items. The serial position effect in comparative judgment is typically a pattern in which pairs that contain a term from one of the two extremes of the list are processed faster and more accurately than pairs that contain no end terms. The experiments show that a new term added to either the end or the middle of a well-practiced fourterm series behaves almost immediately like the end or central term, respectively, of a wellpracticed five-term series. Furthermore, when the added term is removed, the list reverts immediately to the position effect obtained in a four-term series. Theories that explain the position effect by differential build-up of item strength or of interitem associative strength over practice cannot explain these effects. We propose instead that learning of a serial list is accomplished by assigning list members to positions in a general-purpose linear order schema and that subjects can make these assignments rapidly and flexibly.
\end{abstract}

This study is concerned with the structure of the memorial representation that subjects use to process comparative judgments about the relative ordinal position of items in a well-learned serial list. The serial lists examined here give the ordering of a series of objects on some attribute. For example, the "objects" in one of our experiments are fictitious men with names like "Tom," "Dan," and "Ned," and the dimension is height. In this experiment, subjects learn that Tom is taller than Ned, Ned is taller than Dan, and so on. In the comparative judgment testing with the list in this example, subjects are shown pairs from the list and are required to decide, while timed, which name signified the taller or the shorter member of the pair.

Experiments following this general scheme (comparative judgment testing of a short, well-learned list) have consistently shown a number of effects that do not seem to depend on the nature of the attribute used to order the items on the list. These are generally referred to as the semantic congruity effect, the serial position effect, and the distance effect, or, more conveniently, the

This research was supported partly by NSF Grant BNS 7817442 and partly by NIH Grant 1 R01 MH33279-01, both to. W. P. Banks. congruity, position, and distance effects, respectively. These effects are discussed at some length in a number of articles (see, for example, Banks, 1977) and need only brief definitions here. The distance effect is a decline in the time (reaction time, RT) to perform the comparative judgment as the distance between the two members of the list increases.

The congruity effect is an interaction between the term used in the comparative question and the position of the pair on the continuum. The form of the interaction is such that, to refer to the example used above, subjects are relatively faster at picking the taller of two tall athletes than the taller of two short athletes, but they are faster at picking the shorter of two short ones than the shorter of two tall ones. To put it another way, the congruity effect is the condition in which judgments are faster when the instructions are congruent with the items to be judged (i.e., when they name the region of the continuum on which the items fall) than when they are incongruent.

The serial position effect obtained in this paradigm is quite similar in form to the classic serial position effect that has concerned students of human memory over much of the history of the field. The classic position effect is an inverted " $U$," in which end items are easier 
to learn and better remembered than items more central in the list. The serial position effect typically found in comparative judgments shows more errors and longer RTs for judging pairs whose members come from positions central in the list than for pairs whose members come from the end. If we consider pairs a constant distance of one step apart and vary the position in the list from which this pair is taken, a very potent serial position effect usually emerges, in which central pairs can take as much as twice as long to judge as pairs containing end items.

While the position effect for acquisition and retention of single items has occupied researchers and theorists in human memory for some time and has resulted in a very large experimental literature (cf. Crowder, 1976, Chapter 12, for historical and theoretical background), the position effect in the comparative judgment paradigm has not attracted a comparable amount of attention. This is, we think, an unfortunate state of affairs. The position effect offers an arena of experimental test fully as important as that offered by the congruity and distance effects, which seem to have occupied researchers to the exclusion of the position effect.

The distance and congruity effects are related so closely to the position effect that they cannot be analyzed properly without considering the position effect. The distance effect is confounded with the position effect in such a way that under some circumstances it can appear to be much greater than it is unless the position effect is controlled for. In these cases, the confounding has the effect it does because the proportion of pairs in the mean containing the rapidly processed end terms necessarily increases as the span increases. This confounding has been allowed to exist uncontrolled in many distance effects reported in the literature, and the result has been a perhaps unrealistic exaggeration of the importance of the distance effect. If pairs containing terms from the ends of the series are excluded from analysis, the distance effect can be greatly reduced (but may remain statistically reliable) for short series (Potts, 1974) and it can be virtually eliminated for longer series (Moeser, 1979). On the other hand, there are circumstances in which including the end terms in the mean can apparently reduce the distance effect. Such an outcome can occur because the distance effect for pairs containing end terms is often small (cf. Trabasso, Riley, \& Wilson, 1975). Thus, including them in the mean can dilute the overall distance effect.

The congruity effect is closely connected with the position effect. As Banks (1977) noted, the congruity effect for short, overlearned lists can be looked at as a difference in the shapes of the position effects for the two instructions. Both position effects are typically found to be nonmonotonic, but they are asymmetrical, with faster responses at the congruent than at the incongruent end of the continuum. Furthermore, the greatest difference between the two curves will be at the ends of the ordering, so that if pairs containing end terms are excluded from analysis, the congruity effect will typically be much reduced.

All these considerations lead to the conclusion that the position effect deserves the same amount of attention that has been bestowed on the other effects in the paradigm. An understanding of it could yield information on the basis of the distance and congruity effects, as well as on the underlying mental representation of serial orders. This paper provides a critical test of a number of theories of the position effect, these theories being those that assume the position effect results from differential learning of the items at the various positions. These we will term strength theories of the position effect, and they fall into two categories. The first category is simple strength theory. During acquisition, items at the end of the list are learned before those in the middle and thus have more trials of overlearning and more memory strength than central items when the list is finally acquired. The end terms should be identified more accurately and more rapidly than middle terms because of their stronger memory traces; thus, pairs containing at least one end term should be processed in comparative judgment faster and more accurately than pairs containing only terms central in the series.

Another type of strength theory posits, not a unidimensional strength for each item, but various strengths of association among the items in the serial list. The position effect emerges because of a pattern of interitem associative strengths that favors end terms in one way or another. The assumption that learning proceeds by the building up of associations from the end terms has been used to explain the serial position effect in acquisition of lists, tested by memory for individual items (e.g., Glanzer \& Peters, 1962). An analogous assumption of "ends-inward" learning has been made for lists ordered on a dimensional attribute (e.g., DeSoto \& Bosley, 1962; Trabasso \& Riley, 1975).

The present experiments assume that an item added to an already well-learned list should have less memory strength than the original items and should have weaker associations with the other list items than exist among those already in the list. Thus, if the end terms are processed faster because of an advantage in memory strength over the other items in the list, a newly added end term should not be processed faster than any of the older list members and certainly not faster than the original end terms. In the first experiment, subjects first learned a four-term list and made comparative judgments among pairs drawn from the list. Then they had a new term added to one end of the list and were tested again. Finally, the new end term was removed and subjects were given comparative judgments once more on pairs from the original four-term series.

The comparative judgments showed the typical 
serial position effect in the first phase of testing. When the new end term was added, however, the formerly quickly processed end term was processed relatively more slowly than before, and the new term, which could not benefit as much as the others from associative or memory strength, entered into rapidly processed pairs, just as an end term should. The original end term then recovered its end-term character in the third phase of testing when the added term was removed, and it was once more a member of the most rapidly processed pairs. The second experiment uses the same paradigm but adds the new term to the middle of the list. In this experiment, the new term is not processed more quickly than the end terms (and, in fact, behaves much like a midlist term). Thus, the rapid processing of the new end term in the first experiment is unlikely to have resulted from a novelty effect, which would also have accelerated processing to a new middle term in Experiment 2.

We consider the results to be consistent with an account of memory for serial lists whereby the items in the list are associated with abstract place markers in a serial order schema that is applied quite generally to encoding serial orderings. The results indicate that subjects can assign these place markers quite rapidly and flexibly. There are several theories of serial order learning that assume a similar process: Banks (1977), Bower (1971), Ebenholtz (1972), and Wickelgren (1977) all present theories of the representation of serial order that have the ordered items associated with a position in an abstract framework. We do not, however, see the present results as providing specific experimental support for any of these hypotheses. Rather, they only serve to reject a broad class of strength models that might be otherwise entertained as alternatives to these.

\section{EXPERIMENT 1}

\footnotetext{
Method

Subjects. The subjects were 24 students from the Claremont Colleges, paid $\$ 2$ for the approximately $45 \mathrm{~min}$ of the experiment. They were randomly assigned to one of three different groups.

Procedure. The general scheme of this experiment had three phases, in each of which subjects were given a serial list and were then tested with timed comparative judgments of the ordinal rank of pairs drawn from the list. The three groups differed in the nature of the three lists given at each phase. The experimental group was given a four-term list in the first phase, then in the second phase had a new member added, making it a fiveterm list, and then, in the third phase, had the added member removed. This experimental group had two subgroups, one that had the fifth term in the second phase added to the top (unmarked) end, and one that had it added to the bottom.

The other two groups were both control groups. One had the same four-term list throughout the three phases and served as a source of evidence of what would have happened to the experimental groups if the fifth item had not been added in the second phase. The other control group had a single five-term list throughout the three phases. This group allowed a test of whether adding the item in the second phase for the experi-
}

mental group gave results distorted by a novelty (or any other) effect. We will refer to these two groups as the four-term and five-term control groups, respectively.

At the beginning of each phase of the experiment, the subject was given the list appropriate to his or her group and asked to memorize it. The list consisted of either four or five names (Tom, Ned, Bob, Jim, and Dan was the full list from which the terms were drawn), ordered in height. Subjects were allowed an unlimited time for the memorization task, but none took longer than a few minutes. After memorizing was complete in Phase 1 for the control groups and Phases 1 and 2 for the experimental group, subjects were given practice test trials on the experimental task (one block of the 24 or 40 pair-order-question combinations used in the experiment, presented in random order) before the blocks of experimental trials. Brief rest periods were permitted between blocks, and there were 5-min rest periods between phases for all groups. Whenever a name was added to or subtracted from a list, subjects were told whether it was the tallest or shortest member and were then given the revised list to study.

In RT testing, the questions and the pairs of names were presented to subjects on a PDP-11/10 computer-controlled cathode-ray tube display. Subjects began each block by pressing a microswitch; immediately after they pressed it, one of two possible questions, "Taller?" or "Shorter?", appeared for $1 \mathrm{sec}$ on the display, and a pair of names from the list appeared at the end of the $1 \mathrm{sec}$. The subject indicated whether the name appearing on the left or right side of the display was the one specified by the instructions by pressing a microswitch on the left or right side. The subject's response caused the computer to remove the pair of names, present feedback ("correct" or "incorrect"), and begin another stimulus sequence. In each block, the computer program generated a new random order for presentation of the trials. The program recorded RTs for correct responses and number of errors (but not RT for errors) and, later in each block, presented pairs to which responses were incorrect.

In each block of RT testing, all pairs from the list under test were presented once in each permutation and with each instruction. For tests of five-term lists, this procedure created $40 \mathrm{RT}$ trials ( 10 pairs $\times 2$ permutations $\times 2$ instructions), and for fourterm lists, this created 24 trials (6 pairs $\times 2 \times 2$ ). The RT testing part of each phase had six blocks and thus 240 RT trials for the five-term lists and 144 trials for the four-term lists.

\section{Results}

The results for the two experimental groups are shown in Figures 1 and 2. In these figures, the data for the three phases are labeled as pre (i.e., before the added item, or Phase 1), middle (Phase 2), or post (Phase 3). Data for the experimental subgroup in which the new term was added at the top (tallest) end of the series in Phase 2 are shown in Figure 1, and data for the subgroup that had the term added at the bottom of the series are plotted in Figure 2. The stimulus pairs are indicated along the $\mathrm{X}$-axis as hyphenated pairs of numbers that give the ordinal position of the two terms combined in the pair, where 1 is the tallest and 5 is the shortest member of the series. The "new" in parentheses denotes pairs that exist only in the five-term series of Phase 2. In each figure, the leftmost panel plots the data for pairs with an ordinal split of one, the next-to-leftmost plots data for a split of two, the next for a split of three, and the rightmost plots the one mean for the split of four obtained in Phase 2. All points in these 


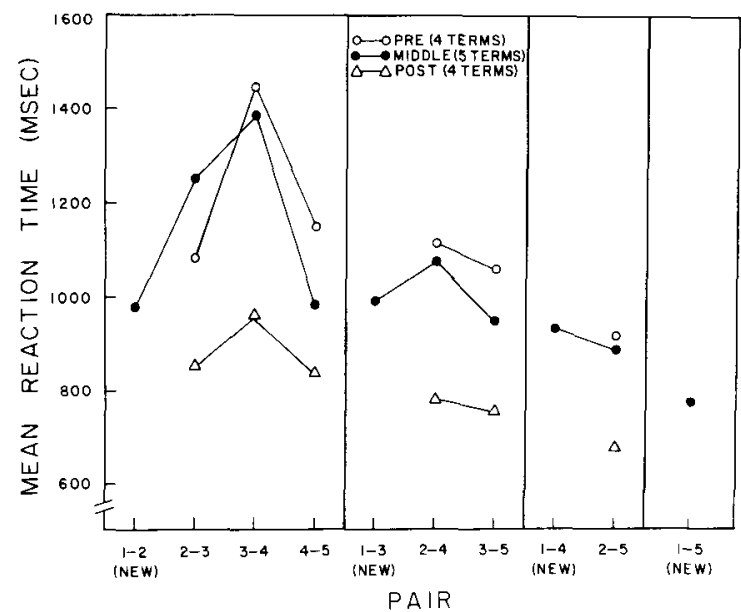

Figure 1. Mean reaction time to make comparative judgments of pairs of men's names drawn from an arbitrary serial ordering. Pairs are designated by the ordinal positions of their members; 1 refers to the tallest member of the series, 2 the next tallest, and so on. Subjects had a series of four names in Phase 1 of the experiment ("pre" in the figure); they then had a new term added to the tall end of the series in Phase 2 ("middle") and had the added term removed in Phase 3 ("post"). Pairs labeled "new" are those that include the new term added in the middle phase.

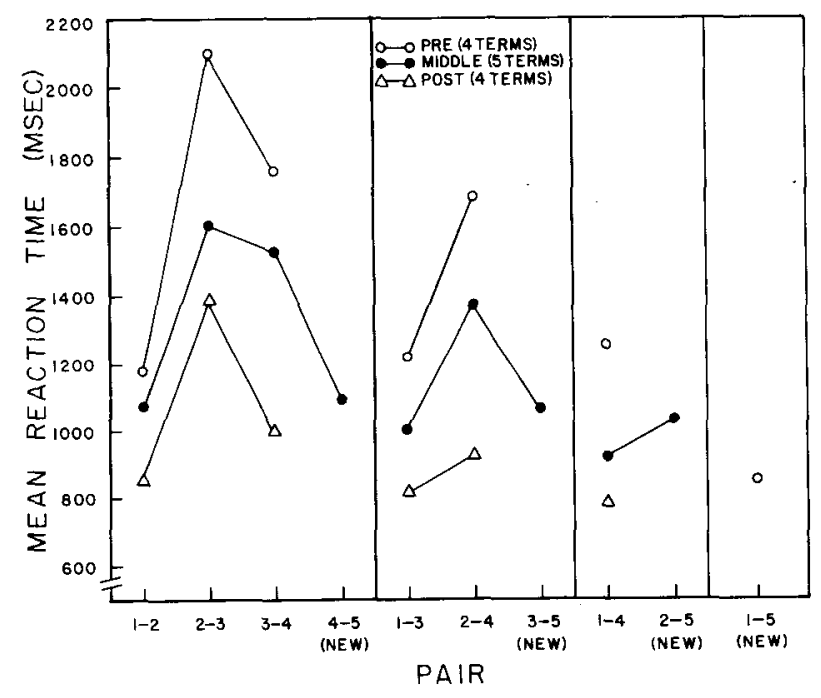

Figure 2. Mean processing time for comparative judgments of serial order of members of pairs, plotted by the same conventions used in Figure 1. Here, however, the term added in the middle phase was put on the short end of the list and is designated as Item 5.

figures represent the mean of the "choose taller" and "choose shorter" instructions.

Several facts are evident in these figures. First, speed of judgment increased over the three phases of the experiment, and second, the bowed serial position effect was maintained in all three. Third, in the middle phase, the added fifth term behaved as an end term should, but the end term from the first phase, relegated to the status of a middle term, behaved like a middle term. In every case, in the middle phase, the pair containing the new end term was judged faster than the comparable pair containing the former end term. Finally, in the third phase of the experiment, the old end term regained its character as an end term when the term added in Phase 2 was dropped.

Two analyses of variance were conducted to assess the reliability of these and other patterns in the data. One analysis used the data from Phases 1 and 3 of both the four-term control group and the experimental group. The other used the data from the middle phase of the five-term control group and the experimental group. Thus, in these analyses, the four-term control group was used only to compare with the two phases of the experimental group's task that had a four-term series and the five-term control to compare with the one experimental phase that had a five-term series. These analyses represent the appropriate use of the control group data and allow an orthogonal analysis of variance.

Both analyses showed reliable differences among the pairs $\left[F(5,35)=18.7, S_{m}=23 \mathrm{msec}, \mathrm{p}<.0001\right.$, for the four-term analysis, and $F(9,63)=16.1, \quad \mathrm{SE}_{\mathrm{m}}=$ $16 \mathrm{msec}, \mathrm{p}<.0001$, for the five-term analysis]. These reliable effects include variance due to both distance and position effects, which will be discussed below. In the four-term analysis, differences between Phases 1 and 3 could be assessed for an increase in judgment speed with practice, which was reliable $[F(1,7)=123.5, p<.0001]$. However, the control and experimental groups did not differ reliably in improvement with practice, and the RTs for individual pairs did not interact with groups or enter into any reliable interactions that included groups (all Fs $<1.0$ ). The unreliability of these effects indicates that adding and then subtracting the fifth term in the middle phase for the experimental group did not have a statistically detectable effect on processing the fourterm series in the final phase. It is as though the fourterm series were reinstated without any aftereffect once the added term is removed.

The comparison between the five-term series in the middle phase of the five-term control and the experimental groups showed no reliable main effects and only one reliable interaction involving group [Pair by Group, $F(9,45)=2.74, p<.025]$. Thus, the subjects who had had a five-term series in the first phase gave, statistically, the same pattern of results in the five-term series of the middle phase as the subjects who had had a four-term series in the first phase and then had one term added to make a five-term series. There was, thus, no evidence of a disruptive effect on the experimental group of adding the term and no evidence that the added term required any time to acquire the status of an end term. Quite the contrary, in fact: although the difference was far from reliable, the experimental group's mean RT for this phase was $1,090 \mathrm{msec}$, compared with the control's $1,133 \mathrm{msec}$. Furthermore, the experimental group showed slightly more pronounced serial position effects than the control group (with the reliable Pair by Group 
interaction). It would appear not only that learning is not required to create the end anchoring in the position effect, but also that the end anchoring may actually decline with practice. However, we did not find a statistically reliable effect of practice on position effects within this phase. While RTs did decline reliably over the six blocks $[F(5,35)=5.6, p<.05]$, the practice effect was statistically parallel over pairs (and, in fact, over all variables and combinations of variables).

The results indicate that the added end item in the middle phase can simply "plug in" to the end position. A long series of trials is definitely not necessary to build up memory strength for the end term. In support of this assertion is the fact that in the very first block of trials with the added item in the experimental group, subjects showed as strong a position effect as they did in the last block. For pairs with splits of one step in Block 1, the difference between the mean of central pairs and pairs containing an end item was $400 \mathrm{msec}$, and on the last block, it was $354 \mathrm{msec}$. For splits of two steps, it was $284 \mathrm{msec}$ on the first block and $114 \mathrm{msec}$ on the last block. If anything, the processing advantage of end terms declines with practice.

It is critically important for the rejection of the memory strength hypothesis that in the middle phase of the experimental group's trials, the new end pair was processed faster than the pair that had been the end pair in Phase 1. For the pairs with a split of one in Figure 1 , the new end pair was 12 in the middle phase and 23 was the original end pair, and, as can be seen, 12 was processed faster. The RT for 12 was $982 \mathrm{msec}$, and it was $1,254 \mathrm{msec}$ for 23 ; this difference was reliable $[F(1,63)=4.56, p<.04]$. The new end pair in the middle phase in Figure 2 is 45 and the old one is 34 , with RTs of $1,094 \mathrm{msec}$ and $1,536 \mathrm{msec}$, respectively $[F(1,63)=12.0, p<.001]$. The pairs with a split of two in Figure 1 are 13 (new end pair) and 24 (original end pair), with RTs of $991 \mathrm{msec}$ and $1,072 \mathrm{msec}$, respectively. In this case, the $F(1,63)$ was less than 1 , but it was reliable for the analogous comparison in Figure 2. These are Pairs 35 (new) and 24 (old), with RTs of $1,065 \mathrm{msec}$ and $1,374 \mathrm{msec}$, respectively $[F(1,63)=$ $5.88, \mathrm{p}<.02]$. Thus, in every case, the pair displaced in the middle phase from its status as an end pair was processed more slowly than the new end pair that displaced it.

Finally, we should consider the congruity and distance effects to see the extent to which they were affected by the position effect. The distance effect in a four-term series was confounded with the position effect, since there was only one pair of the six possible that did not contain an end term. For a series of five, however, it is possible to compare processing times for pairs separated by one and two steps with and without end terms. Looking only at the middle phase of the experimental group (the control group shows the same pattern), we get a difference of $166 \mathrm{msec}$ when pairs with end terms are allowed in the average and a difference of $225 \mathrm{msec}$ when they are not. Thus, the distance effect is actually reduced when the end terms are included. It is interesting to observe the effect of the added end term on the distance effect for the original four-term series in the experimental group. Considering the mean over Phase 1 and Phase 3 (which had four terms), the RTs for ordinal steps of one, two, and three were $1,450 \mathrm{msec}, 1,275 \mathrm{msec}$, and $1,091 \mathrm{msec}$, respectively. These were $1,310 \mathrm{msec}, 1,100 \mathrm{msec}$, and $906 \mathrm{msec}$ for the very same pairs in the middle phase when the fifth term was added. Thus, adding the end term increased the distance effect slightly, from a mean of $179 \mathrm{msec} / \mathrm{step}$ to a mean of $202 \mathrm{msec} / \mathrm{step}$ for the same pairs.

The difference between the congruity effects for the original four-term series with and without the added fifth end term shows how this effect depends on the position effect. The most revealing analysis for this effect looked at the difference in RTs for the two questions at the end of the series to which the term was added in the middle phase. Thus we consider the short end of the series for the subgroup that had the fifth term added there and the top end for the other subgroup. This difference is only half of the total congruity effect, but it is the appropriate difference for this purpose, because the term was added only to one end. In Phases 1 and 3 , for end pairs with a split of one ( 23 for one subgroup and 34 for the other), the mean difference in RT between the two questions was $335 \mathrm{msec}$, and it was $248 \mathrm{msec}$ for the pairs separated by two ordinal steps (24 for one subgroup and 35 for the other). When the fifth end term was added in the middle phase, this difference for the very same pairs became $149 \mathrm{msec}$ for those separated by a split of one and $68 \mathrm{msec}$ for those separated by a split of two. Thus, in one case, this estimate of the size of the congruity effect dropped by $50 \%$, and in the other, by $75 \%$ when the terms in the analysis were displaced from the end of the list.

\section{EXPERIMENT 2}

We interpret the results of Experiment 1 as rejecting the hypothesis that the superior memory strength of end terms is responsible for the fact that pairs containing end terms are processed faster than those not containing end terms. We propose in place of the memory strength hypothesis that each serial position has a code that subjects can rapidly associate with the appropriate item. Before going on to further implications of the results, however, we should consider one possible alternative hypothesis of our results, namely, that the added item in the middle phase benefits from being a new item and is processed quickly by virtue of its novelty. The novelty could serve to overcome the lack of memory strength or to create the advantage for end items that we attribute to structural aspects of the series. Experiment 2 tests the novelty hypothesis by 
adding the fifth term to the middle rather than to the end of the series. A novelty hypothesis predicts that it should enter into rapidly discriminated pairs, just as the added end items in Experiment 1 did. The memory strength hypothesis predicts that pairs containing it should take longer to process than the original ones, and the structural hypothesis predicts that the pairs containing this fifth term should take the same time as the same ones in any five-term series with the equivalent amount of practice.

\section{Method}

This experiment used six female and two male students who were from the same pool used in Experiment 1. The procedure was the same as that for the experimental group in Experiment 1 , except that in the middle phase the name "Dan" was added between Positions 2 and 3 (rather than at the end) of the fourterm list to make the five-term list and, of course, removed for Phase 3 of the experiment.

\section{Results}

The mean RT for each pair is plotted for all three phases in Figure 3, with conventions similar to those observed in Figures 1 and 2. Here, the new term added in the middle phase is Term 3 , and so the pairs that include it are $13,23,34$, and 35 . The appropriate measure of a novelty effect for pairs separated by one step would use the means of Pairs 23 and 34 of the middle phase. It is clear in Figure 3 that neither of these pairs, nor their means, gave a faster response than would be expected. Their means may even be a bit longer than would be expected, juding from the analogous decreases from Phase 1 to Phase 2 in the experimental group of Experiment 1. The mean decrease for the middle pairs

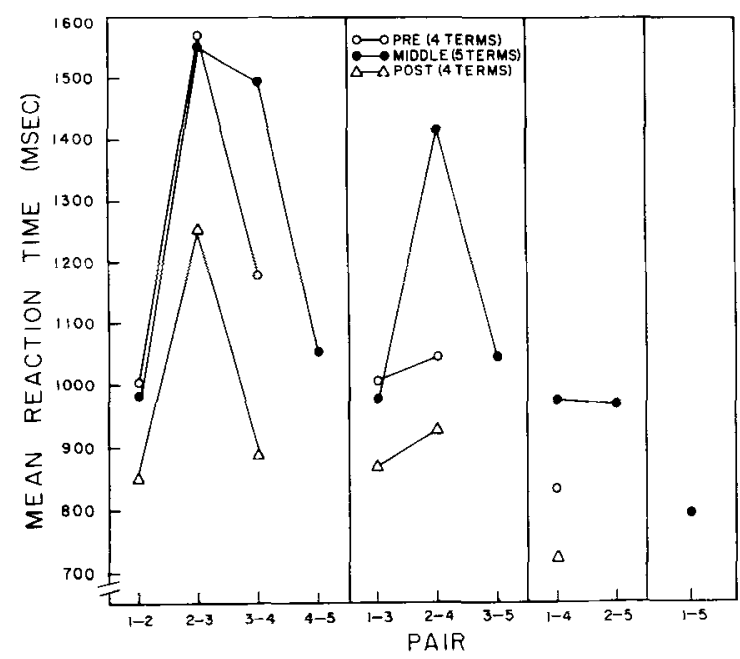

Figure 3. Mean processing time for comparative judgments of serial order of members of pairs, plotted similarly to Figures 1 and 2. Here, the term added in the middle phase was put between Terms 2 and 3 of the four-term series. Thus, in Phase 2 ("middle") the new item is Item 3, and in this middle phase, Items 3 and 4 from Phases 1 and 3 ("pre" and "post") are termed 4 and 5 , respectively.
(34 in Figure 1 and 23 in Figure 2) from Phase 1 to Phase 2 (Pairs 23 and 34) in Experiment 1 was $223 \mathrm{msec}$; here, it was only $44 \mathrm{msec}$.

Judgment times for pairs of terms separated by two steps are shown in the panel second from left in Figure 3. The pairs that include the new term in the middle phase are 13 and 35 , and, as can be seen, these give RTs that fall between those for the comparable pairs for the first and third phases and thus fall where one would expect, given that RTs tend to decrease monotonically with practice. Note what might appear to be a surprisingly long RT for Pair 24 in the middle phase. This pair is, however, the only one with a split of two ordinal steps that does not contain an end term. The fact that it was processed faster than Pairs 23 and 34 at Phase 2 suggests it might have benefited from the additional separation that the inserted term creates in the middle phase.

The next two panels (Pairs 14, 25, and 15) also show longer RTs for the middle phase than one might expect on the basis of the distance effect alone. Pairs 14 and 25 in the middle phase have an ordinal distance of three steps, the same as for Pair 14 for Phases 1 and 3, but the $\mathrm{RT}$ is longer for these pairs in the middle phase. The longer RTs may, however, result from the fact that Pairs 14 and 25 in the middle phase have only one end term, whereas Pair 14 for Phases 1 and 3 has both of them. Pair 15, which exists only in the middle phase, contains the same end terms as Pair 14 for Phases 1 and 3 and yields an RT that falls between the RT for Pair 14 for these two phases. It would appear that the number of end terms in the pair is at least as important in determining RT as the ordinal distance between the members of the pair.

\section{DISCUSSION}

The results of these experiments can be taken as strong evidence against any theory that attributes the serial position effect in comparative judgment of serial order to any incremental process of learning. This includes the "ends-inward" hypothesis of building up memory for a serial list, as well as any sort of associative theory that postulates differential strengths of interitem associations. Likewise, the present results would seem to eliminate any explanation of the faster processing of end terms based on the fact that they are learned first in acquisition trials and hence have more "strength" than the other terms.

To state the conclusions in a different way, we could say that the results indicate that the manner of learning the list does not affect the position effect very much. While we are quite far from sampling every possible procedure of learning a list in this study, the comparison between items in a well-overlearned list and newly learned items added to the list samples extremes of the practice continuum, and yet the position effect seems 
nearly uninfluenced by this difference. Other findings in the literature tend toward the same conclusion. The "conceptual position effect" (Crowder, 1976) is probably the most salient such finding. The conceptual position effect occurs when the end terms are processed faster or more accurately than the middle ones in a conventional ordering, such as the letters of the alphabet or the days of the week, retrieved from semantic memory. It is unlikely that such orderings are learned in the same way (and with the same number of "trials," and so forth) as arbitrary lists in an experiment on memory for serial order, yet both yield the same qualitative function.

The experiments did show practice effects, even though the position effect itself cannot be attributed to them. Mean RT decreased reliably both within and between phases of the experiment, and these practice effects were approximately as strong, in terms of changes in mean RT, as the position effect, measured as the difference between means of central and end terms. The fact that the practice effect was as robust as it was supports the case. against a strength interpretation of the position effect. The large effect of practice on mean RT indicates that the degree of practice was sufficient to yield measurable differences between old and recently added items, if indeed such differences existed. Apparently, practice speeds up RT globally, but differences in degree of practice for individual items in a list cannot account for the position effect. Support for this generalization can be seen very clearly in Figure 1, in which the pair displaced by the new end term in the middle phase actually takes longer to process than it did in the first phase. It seems reasonable to conclude from this and similar findings that the effects of practice and position in the series affect RT by influencing different stages of processing.

What theories of the position effect are not disconfirmed by the present results? First, an interitem associational theory that postulates all-or-none (and virtually instantaneous) learning in this situation could be considered. However, there are a few problems with such a theory. The most important of these is the fact that no theory using interitem associations has yet successfully explained the decline in processing time as members of the pair used in comparative judgment are taken from positions further apart in the list (i.e., the distance effect; Anderson \& Bower, 1973, p. 408; Banks, 1977, pp. 117 118). A lesser problem is the question of the mechanics of the model: If bonds are formed in an all-or-none fashion, how can they have the differential strength needed to predict the differential processing of end vs. middle items that constitutes the position effect? Some theory using all equal-strength bonds could probably be worked out, but we know of none that has survived experimental tests. But, despite our doubts about such a nonincremental interitem associative model, we must admit that the research reported here does not disconfirm it.
The results are consistent with a mental representation of serial order in which the items that are ordered are associated with abstract place markers that signify the part of the ordering to which each item belongs. A number of representations have been suggested that are consistent with our results. The associative models of Trabasso and Riley (1975) and Wickelgren (1977) propose an association between the list items and a position marker, as does the "cognitive scaffold" schema of Bower (1971) or the semantic coding model of Banks (1977). The imagery models of DeSoto, London, and Handel (1965), Huttenlocher (1968), and Moyer (1973) also have the same abstract form, although the representation of ordinal position with which the list items are associated is an analog quantity rather than a mental code for the position in the list.

The main finding of the present study, namely, that new terms can be added to a position in an overlearned serial list and behave immediately as though they had occupied that position in the list all along, indicates that the various effects found in processing of serial lists cannot be ascribed to differential learning of the terms at various positions in the list. Rather, it seems that items can be "plugged" into positions (or removed from them) quite readily, and it is the properties of the positions themselves, or the codes associated with the positions, that determine the processing effects. If differential degrees of incremental memory strength determined the position effect, then new items added to the end should not be processed as quickly as any item already there, and furthermore, the term that the new term displaces as the end term should not lose memory strength because of the displacement. By the same reasoning, interterm associative connections should not suddenly be built up for the new term or destroyed for the displaced term when the new one is added.

\section{REFERENCES}

Anderson, J. R., \& Bower, G. M. Human associative memory. Washington, D.C: Winston, 1973.

BAnks, W. P. Encoding and processing of symbolic information in comparative judgment. In G. H. Bower (Ed.), The psychology of learning and motivation (Vol. 11). New York: Academic Press, 1977.

BowE R, G. Adaptation-level coding of stimuli and serial position effects. In M. H. Appley (Ed.), Adaptation-level theory. New York: Academic Press, 1971.

Crowder, R. G. Principles of learning and memory. Hillsdale, N.J: Eribaum, 1976.

DeSoto, C. B., \& Bosley, J. J. The cognitive structure of a social structure. Journal of Abnormal and Social Psychology, 1962, 64, 303-307.

DeSoto, C. B., London, M., \& Handel, S. Social reasoning and spatial paralogic. Journal of Personality and Social Psychology, 1965, 4, 513-521.

Ebenholtz, S. M. Serial learning and dimensional organization. In G. H. Bower (Ed.), The psychology of learning and motivation (Vol. 5). New York: Academic Press, 1972.

Glanzer, M., \& Peters, S. C. Re-examination of the serial 
position effect. Journal of Experimental Psychology, 1962, 64, 258-266.

HutTenlocher, J. Constructing spatial images: A strategy in reasoning. Psychological Review, 1968, 75, 550-560.

MoESER, S. D. Acquiring complex partial orderings in comparison with acquiring similar-sized linear orderings. Memory \& Cognition, 1979, 7, 435-444.

MOYER, R. S. Comparing objects in memory: Evidence suggesting an internal psychophysics. Perception \& Psychophysics, 1973, 13, 180-184.

PotTs, G. R. Storing and retrieving information about ordered relationships. Journal of Experimental Psychology, 1974, 3, 431-439.
Trabasso, T., \& Riley, C. A. On the construction and use of representations involving linear order. In R. L. Solso (Ed.), Information processing and cognition: The Loyola symposium. Hillsdale, N.J: Erlbaum, 1975.

Trabasso, T., Riley, C. A., \& Wilson, E. G. The representation of linear order and spatial strategies in reasoning: A developmental study. In R. Falmagne (Ed.), Psychological studies of logic and its development. Hillsdale, N.J: Erlbaum, 1975.

Wickelgren, W. A. Cognitive psychology. Englewood Cliffs, N.J: Prentice-Hall, 1977.

(Received for publication March 3, 1980; revision accepted May 21, 1980.) 